This item was submitted to Loughborough's Research Repository by the author.

Items in Figshare are protected by copyright, with all rights reserved, unless otherwise indicated.

\title{
Oophorectomy and hysterectomy may increase dementia risk but only when performed prematurely
}

\section{PLEASE CITE THE PUBLISHED VERSION}

http://dx.doi.org/10.3233/JAD-140909

\section{PUBLISHER}

IOS Press

VERSION

AM (Accepted Manuscript)

\section{PUBLISHER STATEMENT}

This work is made available according to the conditions of the Creative Commons Attribution-NonCommercialNoDerivatives 4.0 International (CC BY-NC-ND 4.0) licence. Full details of this licence are available at: https://creativecommons.org/licenses/by-nc-nd/4.0/

\section{LICENCE}

CC BY-NC-ND 4.0

\section{REPOSITORY RECORD}

Hogervorst, Eef. 2019. "Oophorectomy and Hysterectomy May Increase Dementia Risk but Only When Performed Prematurely". figshare. https://hdl.handle.net/2134/25624. 


\section{Oophorectomy and hysterectomy may increase dementia risk but only when performed prematurely}

Eef Hogervorst Applied Cognitive Research, SSEHS, Loughborough University Loughborough LE11 3TU United Kingdom Tel + 441509223020 Fax + 441509223940 Email: e.hogervorst@lboro.ac.uk

The paper 'Oophorectomy and risk of Alzheimer's disease (AD) among hormone therapy users and non- users' in this edition of JAD describes a very large ( $n=$ almost 40,000) Finnish Nation-wide AD Registered cohort and their age, sex and region-matched controls. In this cohort, oophorectomy for non-malignant indications decreased risk of Alzheimer's disease (AD) by 11-15\%. Hysterectomy alone decreased risk by $12 \%$ and oophorectomy with or without hysterectomy decreased risk by $14 \%$. In a small group of women with bilateral oophorectomy and hysterectomy $(n=92)$ risk was not increased. Risk of AD was independently increased with combined hormone use (HT, estrogen and progesterone) when this was used for less than 10 years. This paper is at odds with a number of other studies in this field, but adds another important piece to the puzzle on whether or not women who undergo surgical menopause should be treated with hormones up to the natural age of menopause to reduce dementia risk.

Several retrospective observational studies suggested that surgical menopause may, when untreated, result in an increased risk for cognitive impairment/dementia risk in later life [1]; [2]; [3]. These studies all found that the earlier the age at surgical menopause with hysterectomy without hormone treatment, the higher the risk of neurological functional decline. Data from the Mayo clinic with a 15 to 52 year follow-up (average 25 years follow-up) reported that oophorectomy for non-malignant reasons $(\mathrm{n}=1472)$ increased the risk of cognitive impairment overall by $46 \%(\mathrm{CI}=1.12-1.90)$ compared to referent women $(\mathrm{n}=1489)$. Oophorectomy before age 38 overall increased this risk by a factor 2.89 $(\mathrm{CI}=1.86-4.48)$ [1]. Women undergoing unilateral oophorectomy were younger and separate analyses of this group showed an overall increased risk of CI by $64 \%$ (CI: 1.20-2.23). When unilateral oophorectomy occurred before age 34, the risk was 4.61 times higher $(\mathrm{CI}=2.52-8.43)$. Before age 41 , this risk was almost a factor 2 higher $(\mathrm{CI}=1.26-3.11)$. This risk seemed dependent on hysterectomy being present, with overall the risk for cognitive impairment co-occurring with any oophorectomy increasing by $1.62(\mathrm{CI}=1.16-2.25)$, and by $1.83(\mathrm{CI}=1.07-3.11)$ for women with unilateral oophorectomy, while this association was only trend significant if the uterus was kept intact.

'Sparing' was thus insufficient, as risk for dementia and Parkinson's disease was increased after untreated unilateral oophorectomy with hysterectomy [4]; [1]. In a small Chinese study [5] 50 elderly women who had undergone unilateral oophorectomy with or without hysterectomy before age at natural menopause were compared to 50 demographically matched controls. Unilateral oophorectomy was associated with worse word recall, often one of the first markers of AD. In a large Danish study (Phung et al., 2010), women born before 1966 and alive on their 40th birthday without a dementia diagnosis prior to $1977(\mathrm{n}=2,313,388)$ were followed from 1977 , or the age of 40, until dementia diagnosis, death, or emigration in 2006. Overall, hysterectomy by itself did not increase the risk of dementia. When stratified by age at dementia diagnoses, hysterectomy was associated with an increased risk for early-onset dementia before the age of 50 which risk increased when accompanied by oophorectomy [hysterectomy alone $(\mathrm{OR}=1.38,95 \%$ confidence interval $(\mathrm{CI})=1.07-1.78)$, with unilateral oophorectomy $(\mathrm{OR}=2.10,95 \% \mathrm{CI}=1.28-3.45)$, and with bilateral oophorectomy $(\mathrm{OR}=2.33,95 \% \mathrm{CI}=1.44-3.77)]$.

However, according to one systematic review (1988 to 2007) this increased risk of dementia after surgical menopause was not always found. The review included a mix of RCT and observational 
studies without analyses of differences in design, methodology and participants included, and often not differentiating whether these women had been treated, whether hysterectomy had also been carried out and for the age at which the procedure had been done ([6], see also the included studies in this review: [7]; [8]; [9]; [10]). Differences in findings between studies could be further related to differences in data collection (self-report or medical records) and design (retro- or prospective data collection, the former being affected by recall). Recall of age at menopause or use of hormones is often impaired long before women develop other clear clinical symptoms of dementia, but this can act as a confound when retrospectively assessing risk factors for dementia [11]. In the present study in $\mathrm{JAD}$, however, hormone use and $\mathrm{AD}$ was assessed through medical records.

A recent US based longitudinal study [3] by Bove et al (2013) included women without dementia at baseline ( $\mathrm{n}=1884$, mean age 78 years at assessment, $\mathrm{n}=607$ had undergone surgical menopause). These participants were from 2 longitudinal studies of cognitive decline: the Religious Orders Study of older Catholic priests, nuns, and brothers that was started in 1994 and the Rush Memory and Aging Project that began enrolling residents of the Chicago area in 1997 who were followed for up to 18 years. Each year of earlier surgical menopause was similar to the cognitive effects associated with 6 months of aging. Age at time of surgical menopause, divided into quartiles (under 38, 38 to 43, 43 to 49 , and over 49 years) was also significant in reducing episodic memory $(P=.0003)$ and semantic memory $(P=.002)$ performance. These tests are both sensitive to early AD and were earlier found to be most responsive to estrogen treatment [12]. An earlier age at surgical menopause was also associated with a higher burden of the global measure of AD neuropathology $(P=.038)$, in particular of neuritic plaques $(P=.013)$. Measures were adjusted for age, education and smoking. In this study there was no association between age at natural menopause and cognition. Unfortunately, no data were available on type of surgery (with or without hysterectomy, unilateral etc).

Several smaller studies also showed that surgical menopause has an acute detrimental effect on cognitive (in particular verbal memory) function in a prospective study of Canadian women (with an average age of 45 years, $n=50$ ) assessed before and after oophorectomy and followed up for 7 months [13]. This was also seen in a cross-sectional study of 103 Italian women with an average age of 51 years [14]. Again, the negative effect on verbal memory was worse when surgery was induced at a younger age [14]. In a prospective 6 month follow-up study of Egyptian women (average age 41 years, $n=53$ ) undergoing surgical menopause resulted in a decline in global cognitive function, whereas controls had stable function over time [15]. However, treatment studies have shown that these effects are reversible.

Randomised controlled trials (RCT) in these early surgical menopausal women (mid to late forties on average) suggested that verbal memory and some other functions (abstract thinking, speed of information processing) showed reversed decline when using high dose intramuscular estradiol or testosterone compared to placebo [13]; [16]; [12]. These findings were highly clinically relevant with one small study showing an average of 10 words more recalled with a high dose intramuscular injection of estradiol or testosterone [13]. Effects on verbal memory were smaller in another RCT from this group but remained stable with estradiol treatment, whereas the placebo group declined [17]

In the observational studies investigating risk for cognitive impairment/dementia, estrogen treatment up to age 50 [1]; or for 10 years within the 5 years around that natural age of menopause [3] eliminated the risk for cognitive impairment and dementia. In the study [3] by Bove et al (2014) of older women, there was no overall significant effect of HT on cognitive decline in this study with 'ever' versus 'never use' or of 'duration of use'. However, duration of HT in this study was associated with slower decline in global cognition when administered within a 5 year perimenopausal window. 
Again, this beneficial effect of hormone treatment in women who had undergone surgical menopause was not always found according to the earlier mentioned systematic review [6]. However, women in the RCTs described in that review [6] who did not respond to hormone treatment, were all older than 50 years of age, and not finding effects of hormone treatment on cognition in that group are conform the later reviews by Rocca and colleagues. Similarly in the present study in JAD, hormone use was not found to modify the associations in this older cohort of postmenopausal women and if anything detrimental associations of combined hormone use with $\mathrm{AD}$ risk were found.

The 'Window of Opportunity theory or Critical Window theory' substantiated by observational and basic sciences data suggests that effects of estrogens are most beneficial when given before or around the natural age at menopause, especially for women undergoing an earlier menopause [18]. Importantly, animal data providing convincing evidence for estradiol's protective effect on brain function also included only ovariectomised animals who responded most significantly when treatment was initiated close to surgery, but not when this interval to treatment initiation was increased [19]. This theory, however, does not explain negative associations of hormone use with cognition.

According to the 'Healthy Cell Bias theory' positive effects of estrogens are seen in healthy neuron cultures, but detrimental effects were found in older neurons already undergoing pathological change [20]. Conform this theory, two observational studies reported that surgical menopausal women who were still using hormone therapy a decade after menopause (around age 60 when they would be more at risk for neuronal pathological changes) actually had worse memory function than those untreated with hormones ([21] $n=36$, aged 51-72 years and [9] $n=885$, aged $60+$ ). Similarly in the present study in JAD, use of hormones was associated with worse risk in this study of older women (average age 81 years). Risk for $\mathrm{AD}$ was slightly decreased in women who had used hormones for more than 10 years which was a small group and may have been those who underwent surgery before the natural age of menopause. In contrast, one very small $(n=35)$ cross-sectional study of women with bilateral oophorectomy (BSO) and hysterectomy aged over 65 years showed that those who were taking hormone treatment performed better on verbal and constructional abilty than non users [22] which would suggest against this theory. However, another much large ( $\mathrm{n}=6110(\mathrm{n}=441 \mathrm{BSO})$, aged 45 to 64 years) study [23] also showed that former, but not current hormone users who had undergone surgical menopause had better verbal fluency (a type of verbal memory test) at a 3 year follow-up than treated and untreated controls who had undergone natural menopause.

Hence, the majority of these studies suggest that hormone treatment up to the age of 50 may be beneficial for neurological function in women who have undergone an early surgical menopause with hysterectomy and that this does not increase risk for dementia. On the other hand, continuing or initiating hormone treatment at an older age ( $>60$ years of age) may confer risk for dementia and accelerate cognitive decline. These data call for a re-evaluation of estrogen treatment for women who become surgically menopausal before age 50 . These data also reflect those of other health related interventions, where cessation of smoking, treating high blood pressure and high cholesterol may also be indicated in early middle age at the latest to reduce the risk of cognitive decline and dementia in later life [24]. It may be that hormone treatment in midlife for a particular subgroup of women (i.e. those who have undergone menopause before the natural average age at menopause) could be part of this, but only for a limited amount of time.

\section{References}

1. Rocca, W.A., et al., Increased risk of cognitive impairment or dementia in women who underwent oophorectomy before menopause. Neurology, 2007. 69(11): p. 1074-83.

2. Phung, T.K., et al., Hysterectomy, oophorectomy and risk of dementia: a nationwide historical 
cohort study. Dement Geriatr Cogn Disord, 2010. 30(1): p. 43-50.

3. Bove, R. and e. al., Age at surgical menopause influences cognitive decline and AD pathology in older women. Neurology, 2013. Dec 11.

4. Rocca, W.A. and e. al., Increased risk of Parkinsonism in women who underwent oophorectomy before menopause. Neurology, 2008. 70(3): p. 200-9.

5. Zhou, G., et al., Cognitive functioning in elderly women who underwent unilateral oophorectomy before menopause. Int J Neurosci, 2011. 121(4): p. 196-200.

6. Vearncombe, K.J. and N.A. Pachana, Is cognitive functioning detrimentally affected after early, induced menopause? Menopause, 2008.

7. Kimura, D., Estrogen replacement therapy may protect against intellectual decline in postmenopausal women. Horm. Behav., 1995. 29: p. 312-321.

8. Kok, H.S., et al., Cognitive function across the life course and the menopausal transition in a British birth cohort. Menopause, 2006. 13(1): p. 19-27.

9. Kritz-Silverstein, D. and E. Barrett-Connor, Hysterectomy, oophorectomy and cognitive function in older women. JAGS, 2002. 50(1): p. 55-61.

10. McLay, R.N., P.M. Maki, and C.G. Lyketsos, Nulliparity and late menopause are associated with decreased cognitive decline. J Neuropsychiatry Clin Neurosci, 2003. 15(2): p. 161-7.

11. Petitti, D.B., et al., Prevalence of dementia in users of hormone replacement therapy as defined by prescription data. J Gerontol A Biol Sci Med Sci, 2002. Aug;57(8): p. M532-8.

12. Hogervorst, E. and S. Bandelow, Sex steroids to maintain cognitive function in women after the menopause: a meta-analyses of treatment trials. Maturitas, 2010. 66(1): p. 56-71.

13. Sherwin, B.B., Estrogen and/or androgen replacement therapy and cognitive functioning in surgically menopausal women. Psychoneuroendocrinology, 1988. 13: p. 345-357.

14. Nappi, R.E., Sinforiani, E., Mauri, M., Bono, G., Polatti, F., Nappi, G., Memory functioning at menopause impact of age in ovariectomized women. Gynecol. Obstet. Invest., 1999. 47: p. 29-36.

15. Farrag, A.K., et al., Effect of surgical menopause on cognitive functions. Dement Geriatr Cogn Disord, 2002. 13(3): p. 193-8.

16. Sherwin, B.B., Estrogenic effects on memory in women. Ann. N.Y. Acad. Sci., 1994. 743: p. 213-230.

17. Phillips, S.M. and B.B. Sherwin, Effects of estrogen on memory function in surgically menopausal women. Psychoneuroendocrinology, 1992. 17: p. 485-495.

18. Hogervorst, E., et al., eds. Hormones, Cognition and Dementia. 2009, Cambridge University Press: Cambridge.

19. Gibbs, R., Animal studies that support estrogen effects on cognitive performance and the cholinergic basis of the critical period hypothesis, in Hormones, Cognition and Dementia, E. Hogervorst, et al., Editors. 2009, Cambridge University Press: Cambridge. p. 45-54.

20. Brinton, R.D., The healthy cell bias of estrogen action. Trends Neuroscience, 2008. 31(10): p. 529-37.

21. File, S.E., J.E. Heard, and J. Rymer, Trough oestradiol levels associated with cognitive impairment in post-menopausal women after 10 years of oestradiol implants. Psychopharmacology (Berl), 2002. 161(1): p. 107-12.

22. Verghese, J., Kuslansky, G., Katz, M., Sliwinsky, M., Crystal, H.A., Lipton, R.B., Surgically menopausal women on estrogen have better cognitive performance. Neurology, 2000. 54(April , Suppl 3): p. A210-A211.

23. Szklo, M., Cerhan, J., Diez-Roux, A.V., Chambless, L., Cooper, L. et al., Estrogen replacement therapy and cognitive functioning in the artherosclerotic risk in communities (ARIC) study. Am. J. Epidemiol., 1996. 144: p. 1048-1057.

24. Clifford, A., et al., Maintaining cognitive health in elderly women: an invited review. Women's Health, 2009. Vol. 5, No. 5, Pages 655-670 
